\title{
Classical quasi-trigonometric $r$-matrices of Cremmer-Gervais type and their quantization
}

\author{
Yermolova-Magnusson J. * \\ Onsala Space Observatory \\ Chalmers University of Technology \\ Göteborg, Sweden \\ Samsonov M. ${ }^{\dagger}$ \\ St. Petersburg State University \\ Institute of Physics \\ St. Petersburg, Russia \\ Stolin A. ${ }^{\ddagger}$ \\ Department of Mathematics \\ University of Göteborg \\ Göteborg, Sweden
}

\begin{abstract}
We propose a method of quantization of certain Lie bialgebra structures on the polynomial Lie algebras related to quasi-trigonometric solutions of the classical Yang-Baxter equation. The method is based on so-called affinization of certain seaweed algebras and their quantum analogues.
\end{abstract}

\section{Introduction}

The aim of this paper is to propose a method of quantizing certain Lie bialgebras structures on polynomial Lie algebras. The subject was introduced by V.G. Drinfeld in the beginning of $90^{\text {th }}$, when in his paper [3] he posed the following problem: can any Lie bialgebra be quantized? Couple of years later, two mathematicians Etingof and Kazhdan came up with the positive answer, while the problem of finding explicit quantization formulas remained open.

*e-mail: julia@oso.chalmers.se

†e-mail: samsonov@pink.phys.spbu.ru

‡e-mail: astolin@math.chalmers.se 
First important contribution to the complete solution of the problem were presented in [11], [4] and [5], where the authors successfully quantized a list of all quasi-triangular Lie bialgebra structures on finite dimensional simple Lie algebras also known as the Belavin-Drinfeld list.

The infinite-dimensional cases were first brought up in [7]. However, a real break-through came with [8] and [12 and was achieved due to construction of a deformed versions of Yangians $Y\left(\mathfrak{s l}_{n}\right)$ and quantum affine algebras $\left.U_{q}(\hat{\mathfrak{s l}})_{n}\right)$ for $n=2,3$.

In the present paper we consider the case $U_{q}\left(\hat{\mathfrak{s l}}_{n}\right)$ for $n=4$. Our solution is based on a $q$-version of so-called seaweed algebras.

A seaweed subalgebra of $\mathfrak{s l}_{n}$ is defined as an intersection of two parabolic subalgebras one of which containing the Borel subalgebra $B^{+}$and another $B^{-}$. The case when both parabolic subalgebras are maximal was treated in connection with the study of rational solutions of the classical Yang-Baxter equation. The results of the study, as well as a complete answer to the question; when such an algebra is Frobenius, can be found in [13]. Later on in [2] it was found out when an arbitrary seaweed algebra is Frobenius.

As a subject of this study we choose a $\mathfrak{s l}_{4}(\mathbb{C}[t])$ Lie algebra which Lie bialgebra structure we are going to quantize. Its coalgebra is defined by a quasi-trigonometric solution of the classical Yang-Baxter equation, this type of solutions was first introduced in [6]. We will begin with a brief summery of the results obtained in this paper.

Let $\mathfrak{g}$ be a simple Lie algebra and let $\Omega \in \mathfrak{g} \otimes \mathfrak{g}$ be the quadratic Casimir element. We say that a solution $X(z, t)$ of the classical Yang-Baxter equation is quasi-trigonometric if it is of the form:

$$
X(z, t)=\frac{t \Omega}{z-t}+p(z, t)
$$

where $p(z, t)$ is a polynomial with coefficients in $\mathfrak{g} \otimes \mathfrak{g}$. Any quasi-trigonometric solution of the classical Yang-Baxter equation defines a Lie bialgebra structure on $\mathfrak{g}[t]$ and the corresponding Lie cobracket on $\mathfrak{g}[t]$ is given by the formula

$$
\{A(t) \in \mathfrak{g}[t]\} \rightarrow\{[X(z, t), A(t) \otimes 1+1 \otimes A(z)] \in \mathfrak{g}[t] \otimes \mathfrak{g}[z]\}
$$

It was proved in [6] that for $\mathfrak{g}=\mathfrak{s l}_{n}$ there is a one-to-one correspondence between quasi-trigonometric $r$-matrices and Lagrangian subalgebras of $\mathfrak{g} \oplus \mathfrak{g}$ transversal to a certain Lagrangian subalgebra of $\mathfrak{g} \oplus \mathfrak{g}$ defined by a maximal parabolic subalgebra of $\mathfrak{g}$. Here we define Lagrangian with respect to the following symmetric non-degenerate invariant bilinear form on $\mathfrak{g} \oplus \mathfrak{g}$ :

$$
Q((a, b),(c, d))=K(a, c)-K(b, d),
$$

where $K$ is the Killing form.

We are going to quantize a quasi-trigonometric $r$-matrix with coefficients in $\mathfrak{s l}_{4} \otimes \mathfrak{s l}_{4}$ defined by the following Lagrangian subalgebra $W$ of $\mathfrak{s l}_{4} \oplus \mathfrak{s l}_{4}$ :

$$
W=\left\{(X, Y) \in \mathfrak{s l}_{4} \oplus \mathfrak{s l}_{4}: X=A d(T) Y\right\} .
$$


Here

$$
T=\left(\begin{array}{llll}
0 & 1 & 0 & 0 \\
1 & 0 & 1 & 0 \\
0 & 0 & 1 & 0 \\
0 & 1 & 0 & 1
\end{array}\right)
$$

\section{Preliminaries}

We denote by $\hat{\mathfrak{s l}}_{n}, n>2$, the following affine Lie algebra with the set of simple affine roots $\left\{\alpha_{0}, \alpha_{1}, \ldots, \alpha_{n-1}\right\}$, defined by the generators $\left\{e_{ \pm \alpha_{i}}, h_{\alpha_{i}}\right\}_{0 \leq i \leq n-1}$ and the relations

$$
\begin{aligned}
{\left[h_{\alpha_{i}}, e_{ \pm \alpha_{j}}\right]= \pm\left(\alpha_{i}, \alpha_{j}\right) e_{ \pm \alpha_{j}}, \quad\left[e_{\alpha_{i}}, e_{-\alpha_{j}}\right]=\delta_{i j} h_{\alpha_{i}} } & \\
e_{ \pm \alpha_{i}}^{2} e_{ \pm \alpha_{j}}-2 e_{ \pm \alpha_{i}} e_{ \pm \alpha_{j}} e_{ \pm \alpha_{i}}+e_{ \pm \alpha_{j}} e_{ \pm \alpha_{i}}^{2}=0, & \text { if }|i-j|=1 ; \\
e_{ \pm \alpha_{i}} e_{ \pm \alpha_{j}}-e_{ \pm \alpha_{j}} e_{ \pm \alpha_{i}}=0, & \text { if }|i-j| \neq 1 .
\end{aligned}
$$

Here $\left\{\left(\alpha_{i}, \alpha_{j}\right)\right\}$ is the affine Cartan matrix of the root system $A_{n-1}^{(1)}$ that is: $\left\{\left(\alpha_{i}, \alpha_{j}\right)\right\}=2$ if $i=j,\left\{\left(\alpha_{i}, \alpha_{j}\right)\right\}=-1$ if $|i-j|=1, n-1$ and 0 otherwise. The root $\alpha_{0}+\alpha_{1}+\ldots+\alpha_{n-1}$ is called imaginary and is denoted by $\delta$, the corresponding Cartan element $h_{\delta}=\sum_{i} h_{\alpha_{i}}$ is central in $\hat{\mathfrak{s l}}_{n}$. It is customary to exclude $\alpha_{0}$ from the defining relations substituting it by $\delta-\alpha_{1}-\ldots-\alpha_{n-1}$.

The universal enveloping algebra $U\left(\hat{\mathfrak{s l}}_{n}\right)$ has the following quantum analogue denoted by $U_{q}\left(\hat{\mathfrak{s l}}_{n}\right)$, which is a Hopf algebra. Formally it is defined by the same set of generators as $U\left(\hat{\mathfrak{s l}}_{n}\right)$, however the defining relations are being $q$-deformed.

$$
\begin{gathered}
{\left[h_{\alpha_{i}}, e_{ \pm \alpha_{j}}\right]= \pm\left(\alpha_{i}, \alpha_{j}\right) e_{ \pm \alpha_{j}}, \quad\left[e_{\alpha_{i}}, e_{-\alpha_{j}}\right]=\delta_{i j} \frac{q^{h_{\alpha_{i}}}-q^{-h_{\alpha_{i}}}}{q-q^{-1}}} \\
e_{ \pm \alpha_{i}}^{2} e_{ \pm \alpha_{j}}-\left(q+q^{-1}\right) e_{ \pm \alpha_{i}} e_{ \pm \alpha_{j}} e_{ \pm \alpha_{i}}+e_{ \pm \alpha_{j}} e_{ \pm \alpha_{i}}^{2}=0, \text { if }|i-j|=1 \\
e_{ \pm \alpha_{i}} e_{ \pm \alpha_{j}}-e_{ \pm \alpha_{j}} e_{ \pm \alpha_{i}}=0, \text { if }|i-j| \neq 1 .
\end{gathered}
$$

A Hopf algebra structure is defined uniquely by the following values of the coproduct on the Chevalley generators

$$
\begin{gathered}
\Delta\left(h_{\alpha_{i}}\right)=h_{\alpha_{i}} \otimes 1+1 \otimes h_{\alpha_{i}} \\
\Delta\left(e_{\alpha_{i}}\right)=q^{-h_{\alpha_{i}}} \otimes e_{\alpha_{i}}+e_{\alpha_{i}} \otimes 1, \quad \Delta\left(e_{-\alpha_{i}}\right)=e_{-\alpha_{i}} \otimes q^{h_{\alpha_{i}}}+1 \otimes e_{-\alpha_{i}} \\
S\left(h_{\alpha_{i}}\right)=-h_{\alpha_{i}}, \quad S\left(e_{\alpha_{i}}\right)=-q^{h_{\alpha_{i}}} e_{\alpha_{i}}, \quad S\left(e_{-\alpha_{i}}\right)=-e_{-\alpha_{i}} q^{-h_{\alpha_{i}}}
\end{gathered}
$$




$$
\varepsilon\left(h_{\alpha_{i}}\right)=0, \quad \varepsilon\left(e_{\alpha_{i}}\right)=0, \quad \varepsilon\left(e_{-\alpha_{i}}\right)=0 .
$$

Following [9, 10], we fix a normal ordering

$$
\begin{aligned}
& \alpha_{1} \prec \alpha_{1}+\alpha_{2} \prec \ldots \prec \alpha_{1}+\alpha_{2}+\ldots+\alpha_{n-1} \prec \alpha_{2} \prec \ldots \prec \alpha_{n-1} \prec \\
& \prec \ldots \prec \delta \prec \ldots \prec \delta-\alpha_{n-1} \prec \delta-\alpha_{n-1}-\alpha_{n-2} \prec \delta-\alpha_{1}-\ldots \prec \alpha_{n-1} .
\end{aligned}
$$

Let $\alpha, \beta$ and $\gamma$ be pairwise non-collinear roots taken so that there are no other roots $\alpha^{\prime}$ and $\beta^{\prime}$ with the property $\gamma=\alpha^{\prime}+\beta^{\prime}$. Then, if $e_{ \pm \alpha}$ and $e_{ \pm \beta}$ have already been constructed, one sets

$$
e_{\gamma}=e_{\alpha} e_{\beta}-q^{(\alpha, \beta)} e_{\beta} e_{\alpha}, \quad e_{-\gamma}=e_{-\beta} e_{-\alpha}-q^{-(\beta, \alpha)} e_{-\alpha} e_{-\beta} .
$$

Consider a seaweed Lie algebra $S W_{n}:=P_{1}^{+} \cap P_{n-1}^{-}$which is defined as the intersection of the maximal parabolic subalgebras $P_{i}^{ \pm}$, obtained by omitting the generators corresponding to the roots containing $\pm \alpha_{i}$. Classically, we have an embedding of the restricted seaweed Lie algebra $\iota: S W_{n}^{\prime} \hookrightarrow \hat{\mathfrak{s l}}_{n-1}$, where

$$
S W_{n}^{\prime}:=\left(S W_{n} \backslash\left\{h_{\alpha_{1}}, h_{\alpha_{n-1}}\right\}\right) \bigcup\left\{p=\sum_{k=1}^{n-1} \frac{(n-2 k)}{n} h_{\alpha_{k}}\right\} .
$$

We show that this embedding can be quantized in the sense that it can be lifted to an embedding:

$$
\iota_{q}: U_{q}\left(S W_{n}^{\prime}\right) \hookrightarrow U_{q}^{\mathcal{K}_{n-1}}\left(\hat{\mathfrak{s l}}_{n-1}\right)
$$

Here the coalgebra structure on $U_{q}\left(S W_{n}^{\prime}\right)$ is obtained from the standard (see above) coalgebra structure on $U_{q}\left(\mathfrak{s l}_{n}\right)$ by twisting it by a certain element $\mathcal{K}_{n} \in$ $U_{q}\left(\mathfrak{s l}_{n}\right)^{\otimes 2}$, which will be described below.

The element $\mathcal{K}_{n}$ will be chosen as follows: $\mathcal{K}_{n}=q^{r_{0}}$, where $r_{0}$ is the Cartan part of the so-called Cremmer-Gervais $r$-matrix. The CremmerGervais $r$-matrices correspond to the shift-by-one Belavin-Drinfeld triples: $\tau: \Gamma_{1} \rightarrow \Gamma_{2}$, where $\Gamma_{i}=\left\{\alpha_{i}, \ldots, \alpha_{n+i-2}\right\}, i=1,2$. To explain this in more details let us recall classification of quasi-triangular $r$-matrices for a simple Lie algebra $\mathfrak{g}$. First of all the quasi-triangular $r$-matrices are solutions of the system

$$
\begin{aligned}
r^{12}+r^{21} & =\Omega \\
{\left[r^{12}, r^{13}\right]+\left[r^{12}, r^{23}\right]+\left[r^{13}, r^{23}\right] } & =0
\end{aligned}
$$

where $\Omega$ is the quadratic Casimir element in $\mathfrak{g} \otimes \mathfrak{g}$.

Belavin and Drinfeld proved that any solution of this system is defined by a triple $\left(\Gamma_{1}, \Gamma_{2}, \tau\right)$, where $\Gamma_{1}, \Gamma_{2}$ are subdiagrams of the Dynkin diagram of $\mathfrak{g}$ and $\tau$ is an isometry between these two subdiagrams. Further, each $\Gamma_{i}$ defines a reductive subalgebra of $\mathfrak{g}$, and $\tau$ extends to an isometry (with respect to the corresponding restrictions of the Killing form) between the obtained two reductive subalgebras of $\mathfrak{g}$. The following property of $\tau$ should be satisfied: $\tau^{k}(\alpha) \notin \Gamma_{1}$ for any $\alpha \in \Gamma_{1}$ and some $k$. Let $\Omega_{0}$ be the Cartan part of $\Omega$. Then one can construct a quasi-triangular $r$-matrix according to the following 
Theorem 1 (Belavin-Drinfeld). Let $r_{0} \in \mathfrak{h} \otimes \mathfrak{h}$ satisfies the systems

$$
\begin{aligned}
r_{0}^{12}+r_{0}^{21} & =\Omega_{0}, \\
(\tau(\alpha) \otimes 1+1 \otimes \alpha)\left(r_{0}\right) & =0
\end{aligned}
$$

for any $\alpha \in \Gamma_{1}$. Then the tensor

$$
r=r_{0}+\sum_{\alpha>0} X_{-\alpha} \otimes X_{\alpha}+\sum_{\alpha, \beta>0, \alpha \succ \beta} X_{-\alpha} \wedge X_{\beta}
$$

satisfies (12), (19). Moreover, any solution of (12), (19) is of the above form, for a suitable triangular decomposition of $\mathfrak{g}$ and suitable choice of a basis $\left\{X_{\alpha}\right\}$.

\section{Quantization of $U_{q}\left(S W_{5}^{\prime}\right)$ and its affinization}

We define $S W_{5} \subset \mathfrak{s l}_{5}$ is the following seaweed Lie subalgebra:

$$
\left(\begin{array}{lllll}
* & 0 & 0 & 0 & 0 \\
* & * & * & * & * \\
* & * & * & * & * \\
* & * & * & * & * \\
0 & 0 & 0 & 0 & *
\end{array}\right) .
$$

Consider the restricted seaweed algebra

$$
S W_{5}^{\prime}:=\left(S W_{5} \backslash\left\{h_{\alpha_{1}}, h_{\alpha_{4}}\right\}\right) \bigcup\left\{p_{5}:=\frac{3}{5} h_{\alpha_{1}}+\frac{1}{5} h_{\alpha_{2}}-\frac{1}{5} h_{\alpha_{3}}-\frac{3}{5} h_{\alpha_{4}}\right\} .
$$

Proposition 1. There exists an embedding: $\iota^{(5)}: S W_{5}^{\prime} \hookrightarrow \hat{\mathfrak{s l}}_{4}$.

Proof. We define $\iota^{(5)}$ by its values on the generators of $S W_{5}^{\prime}$ letting (to avoid ambiguities, we mark the generators of the affine algebra $\mathfrak{s l}_{4}$ by hat, for instance: $\left.\hat{e}_{\alpha}\right)$.

$$
\begin{array}{ll}
\iota^{(5)}\left(p_{5}\right)=\frac{3}{4} h_{\alpha_{1}}+\frac{2}{4} h_{\alpha_{2}}+\frac{1}{4} h_{\alpha_{3}}, & \iota^{(5)}\left(e_{\alpha_{4}}\right)=\hat{e}_{\delta-\alpha_{1}-\alpha_{2}-\alpha_{3}}, \\
\iota^{(5)}\left(e_{-\alpha_{1}}\right)=\hat{e}_{-\alpha_{1}}, & \iota^{(5)}\left(e_{\alpha_{2}}\right)=\hat{e}_{\alpha_{2}}, \\
\iota^{(5)}\left(e_{\alpha_{3}}\right)=\hat{e}_{\alpha_{3}}, & \iota^{(5)}\left(h_{\alpha_{2}}\right)=\hat{h}_{\alpha_{2}}, \\
\iota^{(5)}\left(h_{\alpha_{3}}\right)=\hat{h}_{\alpha_{3}} . &
\end{array}
$$

and fixing the normal ordering in $\hat{\mathfrak{s l}}_{4}$ so that $\alpha_{1} \prec \alpha_{2} \prec \alpha_{3} \prec \delta-\alpha_{1}-\alpha_{2}-\alpha_{3}$. The proof will be finished once we will verify that the defining relations of $S W_{5}^{\prime}$ are being preserved by $\iota^{(5)}$. This is straightforward. 
We define $U_{q}\left(S W_{5}^{\prime}\right)$ as a subalgebra of $U_{q}\left(\mathfrak{s l}_{5}\right)$ generated by $h_{\alpha_{2}}, h_{\alpha_{3}}, p_{5}$, $e_{-\alpha_{1}}, e_{\alpha_{4}}, e_{ \pm \alpha_{2}}, e_{ \pm \alpha_{3}}$. Unfortunately, the subalgebra defined as $U_{q}\left(S W_{5}^{\prime}\right)$ is not a Hopf subalgebra of $U_{q}\left(\mathfrak{s l}_{5}\right)$ with respect to the standard comultiplication in $U_{q}\left(\mathfrak{s l}_{5}\right)$. However, twisting the standard comultiplication by $\mathcal{K}_{5}=q^{r_{0}(5)}$, where $r_{0}(5)$ is the Cartan part of the Cremmer-Gervais $r$-matrix for $\mathfrak{s l}_{5}$, we obtain the following result:

Proposition 2. Let $\mathcal{K}_{5}=q^{r_{0}(5)}$ where

$$
\begin{aligned}
& r_{0}(5)= \\
& h_{\alpha_{1}} \otimes\left(\frac{2}{5} h_{\alpha_{1}}+\frac{3}{5} h_{\alpha_{2}}+\frac{3}{5} h_{\alpha_{3}}+\frac{2}{5} h_{\alpha_{4}}\right)+h_{\alpha_{2}} \otimes\left(\frac{3}{5} h_{\alpha_{2}}+\frac{4}{5} h_{\alpha_{3}}+\frac{3}{5} h_{\alpha_{4}}\right)+ \\
& +h_{\alpha_{3}} \otimes\left(-\frac{1}{5} h_{\alpha_{1}}+\frac{3}{5} h_{\alpha_{3}}+\frac{3}{5} h_{\alpha_{4}}\right)+h_{\alpha_{4}} \otimes\left(-\frac{1}{5} h_{\alpha_{1}}-\frac{1}{5} h_{\alpha_{2}}+\frac{2}{5} h_{\alpha_{4}}\right)
\end{aligned}
$$

is the Cartan part of the Cremmer-Gervais r-matrix. Then the Hopf algebra $U_{q}^{\mathcal{K}_{5}}\left(\mathfrak{s l}_{5}\right)$ induces a coalgebraic structure on the quantum seaweed algebra $U\left(S W_{5}^{\prime}\right) \subset U_{q}^{\mathcal{K}_{5}}\left(\mathfrak{s l}_{5}\right)$.

Proof. Let us consider $U_{q}^{\mathcal{K}_{5}}\left(\mathfrak{s l}_{5}\right)$ and check that the elements corresponding to the generators of $U_{q}\left(S W_{5}^{\prime}\right)$ define a Hopf subalgebra in $U_{q}^{\mathcal{K}_{5}}\left(\mathfrak{s l}_{5}\right)$. In order to prove this, we have to show that the following relations hold for $1 \leq i \leq 4$ :

$$
\begin{aligned}
\left(\alpha_{i} \otimes \mathrm{id}\right)\left(r_{0}(5)\right) & \in U_{q}\left(S W_{5}^{\prime}\right) \\
\left.-h_{\alpha_{i}}+\left(\mathrm{id} \otimes \alpha_{i}\right)\left(r_{0}(5)\right)\right) & \in U_{q}\left(S W_{5}^{\prime}\right) \\
\left(\mathrm{id} \otimes \alpha_{i}\right)\left(r_{0}(5)\right) & \in U_{q}\left(S W_{5}^{\prime}\right) \\
h_{\alpha_{i}}+\left(\alpha_{i} \otimes \mathrm{id}\right)\left(r_{0}(5)\right) & \in U_{q}\left(S W_{5}^{\prime}\right) .
\end{aligned}
$$

Taking into account that $h_{\alpha_{2}}, h_{\alpha_{3}} \in U_{q}\left(S W_{5}^{\prime}\right)$ and formulas (14)-(15), we see that using the explicit formula for $r_{0}(5)$ it is sufficient to prove that

$$
\left(\mathrm{id} \otimes \alpha_{1}\right)\left(r_{0}(5)\right)=\frac{1}{5} h_{\alpha_{1}}-\frac{3}{5} h_{\alpha_{2}}-\frac{2}{5} h_{\alpha_{3}}-\frac{1}{5} h_{\alpha_{4}} \in U_{q}\left(S W_{5}^{\prime}\right) .
$$

It can be checked directly and this finishes the proof.

In what follows we need to introduce an element $\hat{\mathcal{K}}_{4} \in U_{q}\left(\hat{\mathfrak{s l}}_{4}\right)^{\otimes 2}$, which is a four-dimensional analogue of the element $\mathcal{K}_{5} \in U_{q}\left(\mathfrak{s l}_{5}\right)^{\otimes 2}$ :

$\hat{\mathcal{K}}_{4}=q^{\hat{r}_{0}(4)}$, where

$$
\begin{aligned}
& \hat{r}_{0}(4)= \\
& \hat{h}_{\alpha_{1}} \otimes\left(\frac{3}{8} \hat{h}_{\alpha_{1}}+\frac{1}{2} \hat{h}_{\alpha_{2}}+\frac{3}{8} \hat{h}_{\alpha_{3}}\right)+\hat{h}_{\alpha_{2}} \otimes\left(\frac{1}{2} \hat{h}_{\alpha_{2}}+\frac{1}{2} \hat{h}_{\alpha_{3}}\right)+\hat{h}_{\alpha_{3}} \otimes\left(-\frac{1}{8} \hat{h}_{\alpha_{1}}+\frac{3}{8} \hat{h}_{\alpha_{3}}\right) .
\end{aligned}
$$

Theorem 2. There is an embedding of Hopf algebras:

$$
\iota_{q}^{(5)}: U_{q}\left(S W_{5}^{\prime}\right) \hookrightarrow U_{q}^{\hat{\mathcal{K}}_{4}}\left(\hat{\mathfrak{s l}}_{4}\right)
$$

where $\iota_{q}^{(5)}$ is defined by the same formulas on the quantum generators as $\iota^{(5)}$ was defined on the classical generators in Proposition 1. 
Proof. By the properties (12)-(13) it is sufficient to verify that

$$
\begin{aligned}
& \iota_{q}^{(5)}\left(\left(\operatorname{id} \otimes \alpha_{1}\right)\left(r_{0}(5)\right)\right)=\left(\mathrm{id} \otimes \alpha_{1}\right)\left(\hat{r}_{0}(4)\right) \\
& \iota_{q}^{(5)}\left(\left(\alpha_{4} \otimes \mathrm{id}\right)\left(r_{0}(5)\right)\right)=-\left(\left(\alpha_{1}+\alpha_{2}+\alpha_{3}\right) \otimes \mathrm{id}\right)\left(\hat{r}_{0}(4)\right)
\end{aligned}
$$

(21) is equivalent to the following equality

$$
\iota_{q}^{(5)}\left(\frac{1}{5} h_{\alpha_{1}}-\frac{3}{5} h_{\alpha_{2}}-\frac{2}{5} h_{\alpha_{3}}-\frac{1}{5} h_{\alpha_{4}}\right)=\frac{1}{4} \hat{h}_{\alpha_{1}}-\frac{1}{2} \hat{h}_{\alpha_{2}}-\frac{1}{4} \hat{h}_{\alpha_{3}}
$$

while (22) can be rewritten as follows:

$$
\iota_{q}^{(5)}\left(-\frac{1}{5} h_{\alpha_{1}}-\frac{2}{5} h_{\alpha_{2}}-\frac{2}{3} h_{\alpha_{3}}+\frac{1}{5} h_{\alpha_{4}}\right)=-\frac{1}{4} \hat{h}_{\alpha_{1}}-\frac{1}{2} \hat{h}_{\alpha_{2}}-\frac{3}{4} \hat{h}_{\alpha_{3}} .
$$

\section{Quantization of quasi-trigonometric $r-$ ma- trices}

Following [4, 5], we can construct the Cremmer-Gervais twist in $U_{q}\left(\mathfrak{s l}_{5}\right)$ as the following product:

$$
\mathcal{F}_{C G_{5}}=\mathcal{F}^{(3)} \mathcal{F}^{(2)} \mathcal{F}^{(1)} \cdot \mathcal{K}_{5}
$$

where

$$
\begin{gathered}
\mathcal{F}^{(k)}=\prod_{\alpha_{1} \preceq \beta \preceq \alpha_{3}} \exp _{q^{2}}\left(\left(q^{-1}-q\right) e_{\tau^{k}(\beta)}^{\prime} \otimes e_{-\beta}^{\prime}\right), \\
\exp _{q}(x):=\sum_{n \geq 0} \frac{x^{n}}{(n)_{q} !}, \quad(n)_{q} ! \equiv(1)_{q}(2)_{q} \ldots(n)_{q}, \quad(k)_{q} \equiv\left(1-q^{k}\right) /(1-q)
\end{gathered}
$$

and

$$
e_{\alpha_{k}}^{\prime}=q^{\left(\alpha_{k} \otimes \mathrm{id}\right)\left(r_{0}(5)\right)} e_{\alpha_{k}}, \quad e_{-\alpha_{k}}^{\prime}=q^{-\left(\mathrm{id} \otimes \alpha_{k}\right)\left(r_{(0)}(5)\right)} e_{-\alpha_{k}} .
$$

The elements $e_{ \pm \alpha_{k}}^{\prime}$ have the following coproducts after twisting by $\mathcal{K}_{5}$ :

$$
\begin{aligned}
& \mathcal{K}_{5} \Delta\left(e_{\alpha_{k}}^{\prime}\right) \mathcal{K}_{5}^{-1}=e_{\alpha_{k}}^{\prime} \otimes q^{2\left(\alpha_{k} \otimes \mathrm{id}\right)\left(r_{0}(5)\right)}+1 \otimes e_{\alpha_{k}}^{\prime} \\
& \mathcal{K}_{5} \Delta\left(e_{-\alpha_{k}}^{\prime}\right) \mathcal{K}_{5}^{-1}=q^{-2\left(\mathrm{id} \otimes \alpha_{k}\right)\left(r_{0}(5)\right)} \otimes e_{-\alpha_{k}}^{\prime}+e_{-\alpha_{k}}^{\prime} \otimes 1 .
\end{aligned}
$$

Corollary 1. $\mathcal{F}_{C G_{5}}^{\prime}=\mathcal{F}_{C G_{5}} \mathcal{K}_{5}^{-1}$ restricts to a twist of $U_{q}\left(S W_{5}^{\prime}\right)$ leading to an affine twist in $U_{q}\left(\hat{\mathfrak{s} \mathfrak{l}_{4}}\right)$ via embedding $\iota_{q}^{(5)}$ :

$$
\hat{\mathcal{F}}_{C G_{4}}=\left(\iota_{q}^{(5)} \otimes \iota_{q}^{(5)}\right)\left(\mathcal{F}_{C G_{5}} \cdot \mathcal{K}_{5}^{-1}\right) \cdot \hat{\mathcal{K}}_{4} .
$$

Proof. Since the element $\mathcal{K}_{5}$ is a twist for $U_{q}\left(\mathfrak{s l}_{5}\right)$ we see that:

$$
\mathcal{K}_{5}^{12}(\Delta \otimes \mathrm{id})\left(\mathcal{K}_{5}\right)=\mathcal{K}_{5}^{23}(\mathrm{id} \otimes \Delta)\left(\mathcal{K}_{5}\right),
$$

then, by the chain property of the twists, $\mathcal{F}_{C G_{5}}^{\prime}$ defines a twist for $U_{q}^{\mathcal{K}_{5}}\left(\mathfrak{s l}_{5}\right)$, and it can be restricted to a twist for $U_{q}\left(S W_{5}^{\prime}\right)$ leading to (23) if we use the same argument relating $U_{q}\left(\hat{\mathfrak{s}}_{4}\right)$ and $U_{q}^{\hat{\mathcal{K}}_{4}}\left(\hat{\mathfrak{s}}_{4}\right)$ via twisting by $\hat{\mathcal{K}}_{4}$. 


\section{Discussion}

It follows from Proposition 1 that in the present paper we have quantized the following quasi-trigonometric $r$-matrix in $\mathfrak{s l}_{4}$ :

$$
\begin{aligned}
& X(z, t)=\frac{t \Omega}{z-t}+r_{D J}+ \\
& S K\left(\left(e_{23}+e_{34}\right) \otimes e_{21}+e_{24} \otimes e_{31}+e_{34} \otimes e_{32}-r_{0}(4)+\right. \\
& (z-t)\left(e_{21} \otimes e_{41}+e_{41} \otimes e_{21}+e_{31} \otimes e_{31}\right)+ \\
& z e_{31} \otimes e_{42}-t e_{42} \otimes e_{31}+z e_{41} \otimes e_{32}-t e_{32} \otimes e_{41} .
\end{aligned}
$$

Here $e_{i j}$ are matrix units, $S K(a \otimes b)=a \otimes b-b \otimes a$ and $r_{D J}$ is the DrinfeldJimbo $r$-matrix for $\mathfrak{s l}_{4}$ In terms of Theorem 1 it is defined by the following data:

$$
\Gamma_{1}=\Gamma_{2}=\emptyset, \quad r_{0}=\frac{\Omega_{0}}{2}
$$

The corresponding quantum algebra can be obtained by twisting the standard quantum affine algebra $U_{q}\left(\hat{\mathfrak{s l}}_{4}\right)$ by the element $\hat{\mathcal{F}}_{C G_{4}}$. Then the quan-

tum $R$-matrix $\hat{\mathcal{F}}_{C G_{4}}^{21} R_{a f f} \hat{\mathcal{F}}_{C G_{4}}^{-1}$ quantizes $X(z, t)$. The universal quantum $R$-matrix $R_{a f f}$ was constructed in [10].

Using methods of [6] one can prove that this $r$-matrix is gauge equivalent to that of defined by the Lagrangian subalgebra $W \subset \mathfrak{s l}_{4} \oplus \mathfrak{s l}_{4}$ from Introduction. Extra terms of $X(z, t)$,

$$
X(z, t)-\frac{t \Omega}{z-t}-r_{D J}
$$

define a classical twist for the polynomial Lie bialgebra $\mathfrak{s l}_{4}[t]$.

As a conclusion, we've to mention that the results presented in this paper support the conjecture made in [6], namely:

every classical twist gives rise to a quantum twist.

\section{References}

[1] Cremmer, E. and Gervais, J.L.: The quantum group structure associated with nonlineary extended Virasoro algebras, Comm. Math. Phys. 134(3)(1990), 619-632.

[2] Dergachev, V., and Kirillov, A.: Index of Lie algebras of seaweed type. $J$. Lie Theory 10(2) (2000), 331-343.

[3] Drinfeld, V. G.: On some unsolved problems in quantum group theory. Quantum groups (Leningrad, 1990), 1-8, Lecture Notes in Math., 1510, Springer, Berlin, 1992. 
[4] Etingof, P., Schedler, T. and Schiffmann: Explicit quantization of dynamical $r$-matrices for finite dimensional semisimple Lie algebras, J. Amer. Math. Soc. 13 (2000), 595-609.

[5] Isaev, A.P. and Ogievetsky, O.V.: On Quantization of $r$-matrices for Belavin-Drinfeld Triples, Physics of Atomic Nuclei 64(12) (2001), 21262130, math.QA/0010190.

[6] Khoroshkin, S.M., Pop, I.I., Stolin, A.A. and Tolstoy, V.N.: On some Lie bialgebra structures on polynomial algebras and their quantization, preprint, Mittag-Leffler Institute, Sweden, 2004.

[7] Khoroshkin, S. M., Stolin, A. A. and Tolstoy, V. N.: Deformation of Yangian $Y\left(\mathrm{sl}_{2}\right)$. Comm. Algebra 26(4) (1998), 1041-1055.

[8] Khoroshkin, S. M., Stolin, A. A. and Tolstoy, V. N.: $q$-power function over $q$-commuting variables and deformed $X X X$ and $X X Z$ chains, Phys. Atomic Nuclei 64(12) (2001), 2173-2178; translated from Yadernaya Fiz. 64(12) (2001), 2262-2267.

[9] Khoroshkin, S. and Tolstoy, V.: Universal $R$-matrix for quantized superalgebras, Comm. Math. Phys. 141(3) (1991), 599-617.

[10] Tolstoy, V. N. and Khoroshkin, S. M.: Universal $R$-matrix for quantized nontwisted affine Lie algebras. (Russian) Funktsional. Anal. i Prilozhen. 26(1) (1992), 85-88.

[11] Kulish, P. P. and Mudrov, A. I.: Universal $R$-matrix for esoteric quantum groups. Lett. Math. Phys. 47(2)(1999), 139-148.

[12] Samsonov, M.: Semi-classical Twists for $\mathfrak{s l}_{3}$ and $\mathfrak{s l}_{4}$ Boundary $r$-matrices of Cremmer-Gervais type, Lett. Math. Phys., 72(3)(2005), 197-210.

[13] Stolin, A.: On rational solutions of Yang-Baxter equation for $\mathfrak{s l}(n)$, Math. Scand. 69(1) (1991), 57-80. 